
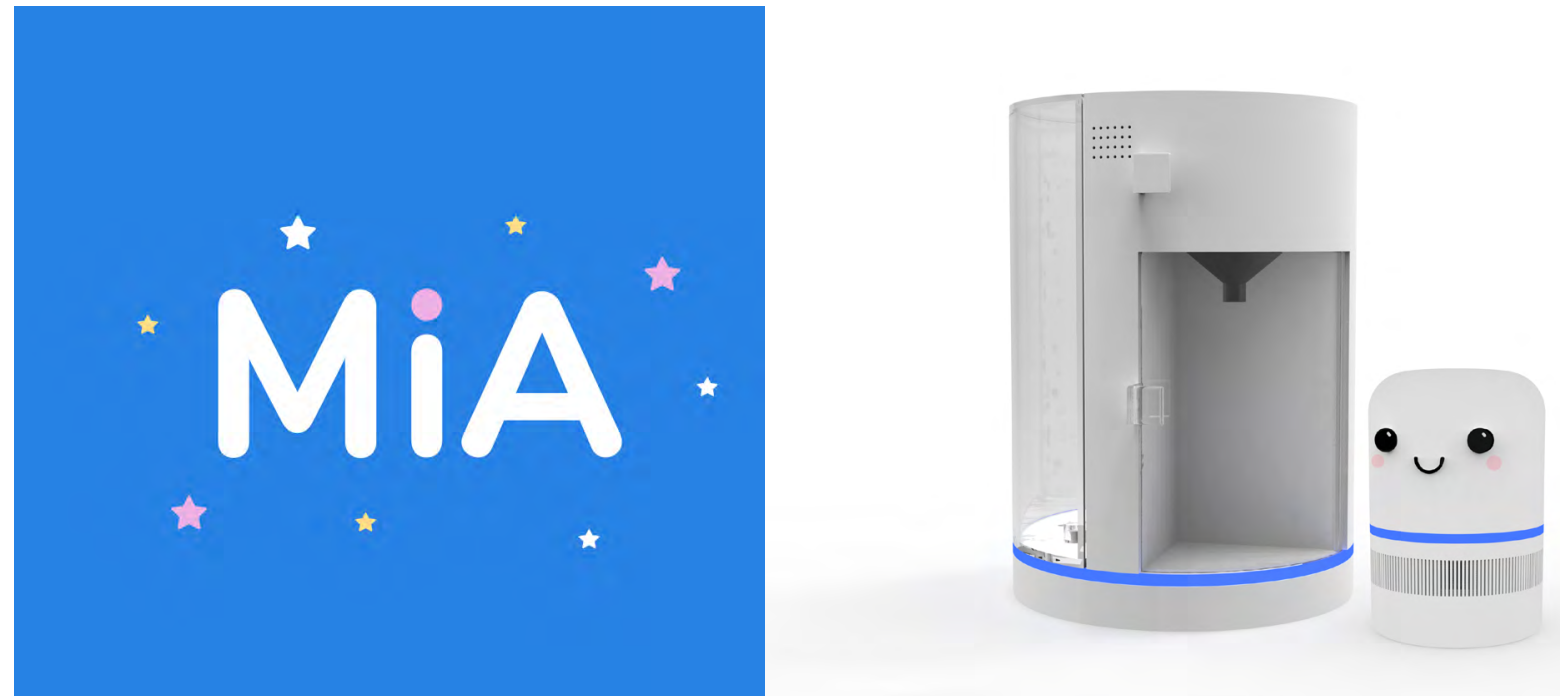

\title{
MiA: Diseño y prototipado de un sistema de asistencia para la maternidad
}

MiA: Designing and prototyping a maternity assistant system

\author{
María Fernanda Arce-Murillo ${ }^{1}$, Sara Yariela Robles-Sandoval ${ }^{2}$, \\ Ariel Rojas-Monge ${ }^{3}$, Hilda Vásquez-Carvajal ${ }^{4}$
}

M.F. Arce-Murillo, S.Y. Robles-Sandoval, A. Rojas-Monge, and H. VásquezCarvajal, "MiA: Diseño y prototipado de un sistema de asistencia para la maternidad", IDI+, vol. 3, no 1, Jul., pp. 4-16, 2020.

doi https://doi.org/10.18845/ridip.v3i1.5305

Fecha de recepción: 18 de noviembre 2019 Fecha de aprobación: 29 de mayo de 2020

1. María Fernanda Arce-Murillo Estudiante de Ingeniería en Diseño Industrial Instituto Tecnológico de Costa Rica Cartago, Costa Rica

fernanda.am1863@gmail.com (iD 0000-0003-2481-0289

2. Sara Yariela Robles-Sandoval Estudiante de Ingeniería en Diseño Industrial Instituto Tecnológico de Costa Rica Cartago, Costa Rica sararobless1998@gmail.com (iD 0000-0002-1385-3454
3. Ariel Rojas-Monge Estudiante de Ingeniería en Diseño Industrial Instituto Tecnológico de Costa Rica Cartago, Costa Rica armsp10@gmail.com (iD) 0000-0002-9025-9897

4. Hilda Vásquez-Carvajal Estudiante de Ingeniería en Diseño Industrial Instituto Tecnológico de Costa Rica Cartago, Costa Rica

hildarvc16@gmail.com (D) 0000-0003-2076-9207 


\section{Resumen}

En el marco del mercado actual, donde las personas se encuentran en una búsqueda constante de herramientas para facilitar su vida, se decidió desarrollar un sistema de objetos inteligentes que ayuden en las tareas de la maternidad. Al inicio del proyecto, se estudiaron usuarios con la característica de "paternidad reciente", para determinar así las necesidades más relevantes y las tareas que a estas acompañaban. Tras el conocimiento de las necesidades, se sintetizaron los problemas: preparar biberones de manera eficiente y vigilar al infante por un breve periodo. Seguido de estos hallazgos, se planteó un concepto de diseño, con el cual se esbozaron propuestas de solución que posteriormente se evaluaron con las necesidades determinadas hasta llegar a la que mejor satisficiera los requisitos. Esta propuesta se llevó a un prototipo de baja fidelidad por medio de impresión 3D, además, se probó su funcionalidad y usabilidad con usuarios reales, para así tener el resultado final.

\section{Palabras Clave}

Objeto inteligente; maternidad; diseño industrial; asistente; sistema.

\section{Abstract}

In the framework of the current market, where people are constantly searching for tools to facilitate their lives, we developed a system of intelligent objects to help in motherhood tasks.

To start the project, subjects with the "recent parent-hood" characteristic were studied to determine the most outstanding necessities and duties that accompany this circumstance. After acknowledging these necessities, the problems were synthesized: efficiently preparing baby bottles, and watching after the infant for a brief period.

Following these findings, the designing concept was stated; based on it, the solution sketches were drawn, and evaluated with the necessities determined, until the one that met the criteria the most was found. Then, it was high fidelity-prototyped via 3D printing and finally tested with real users to validate the final product.

\section{Keywords}

Smart object; parenting; industrial design; assistant; system. 


\section{Introducción}

Ser padres, primerizos o experimentados, no es tarea fácil, y con el nacimiento de un bebé se tiende a complicar la situación. Comienzan a haber problemas como descuidos, falta de sueño, cansancio mental y físico, llanto del niño, frustración y otros, que causan un agotamiento en los padres y pueden convertir la experiencia en un caos.

\begin{tabular}{l|c} 
Total & $\mathbf{6 8 4 7 9}$ \\
\hline San José & \\
\hline Alajuela & 19843 \\
\hline Cartago & 15342 \\
\hline Heredia & 6710 \\
\hline Guanacaste & 6155 \\
\hline Puntarenas & 5629 \\
\hline Limón & 7344 \\
\hline
\end{tabular}

Figura 1. Tabla de nacimientos por provincia en el 2018.

Fuente: INEC-Costa Rica, Unidad de Estadísticas Demográficas, 2018.

De acuerdo con los datos de la figura 1, se puede apreciar que, solo en Costa Rica, hubo un total de 68479 bebés nacidos en el 2018 y en el primer semestre del 2019, un total de 31 801 [1]; así mismo, cada vez son más las madres que toman la alternativa de alimentar a su bebé con biberón en vez de amamantar [2], lo que implica una posibilidad de impactar a más de 30000 usuarios potenciales por año, solo a nivel nacional. Esto significa que hay un amplio mercado para el consumo de los productos para la maternidad.

Dentro de estos productos que se encuentran disponibles en el mercado, los más utilizados son los monitores maternos, los cuales ayudan a la vigilancia del bebé, así como los calentadores de biberones, que tienen la capacidad de calentar un biberón de manera más eficiente. Además, en la última década, se ha dado el lanzamiento de un sinfín de productos inteligentes, como smartphones, parlantes inteligentes, lavadoras, bombillos y otros más, que simplifican y alivianan la carga a los usuarios, de una manera que sus homólogos analógicos no hacen [3].

Por estos motivos, se plantea la creación de un sistema de productos (Maternity Intelligent Assistant) que permita integrarse en este entorno; el Smart Formula Maker (SFM) y el Smart Nanny (SN). Estos productos se encargan, respectivamente, de preparar biberones y de monitorear al niño por un breve periodo. 
EI SFM cuenta con reconocimiento de voz para poder interactuar con este a distancia, ya que muestra ser la manera de control preferida por los usuarios [4]; también posee la capacidad de programar la preparación de biberones, se comunica con el SN, puede leer la temperatura del biberón y enviar notificaciones vía SMS al celular. Por otra parte, el SN tiene un sensor de movimiento de $360^{\circ}$, emisión de luz, emisión de sonido, así como reconocimiento de voz, la capacidad de notificar vía SMS al celular y comunicarse con el SFM.

\section{Metodología}

En este caso, se realizaron seis etapas que abarcaban los pasos básicos del proceso proyectual para que la comprensión de los resultados fuera más fluida y sencilla. Dichos pasos se describen a continuación.

\section{Conceptualizando la idea}

Primero, se estudiaron los distintos escenarios de uso, así como los posibles usuarios y sus contextos de uso. El usuario ideal son padres primerizos o que tuvieran bebés menores a 18 meses y con un estilo de vida que involucre el uso de tecnología inteligente. Con base en los datos proporcionados por el INEC, se evidenció que hubo un total de 68479 nacimientos en el año 2018 en Costa Rica, también se observó que, en promedio, cada costarricense tiene 1.67 hijos, es decir, que gran cantidad de personas, alrededor de 25 000, son padres primerizos, por lo tanto, calzarían con el mercado meta del producto y, si tienen experiencia utilizando tecnologías inteligentes (i.e. Alexa, Google Home, etc.), serían el usuario ideal.

Seguido a esto, se entrevistó a cinco personas que coincidían con los usuarios determinados en el paso anterior. Se realizó una guía para la entrevista basada en los datos de relevancia que se necesitaban para el proyecto. Además, se acordó una cita con cada uno de los usuarios para conversar y grabar sus respuestas con el permiso de cada uno. Con los datos recopilados, se extrajeron las necesidades más importantes y contundentes para solucionar por medio de tecnología inteligente [5], a través de un árbol de problemas; esto se sintetizó en una lista de requisitos y requerimientos que permitieron parametrizar y ponderar la importancia de las diferentes necesidades.

Luego, se realizó un estudio de mercado con el uso de datos del INEC-Costa Rica del 2018 y del primer semestre del 2019, así como un análisis de lo existente, con lo que se comparó la manera en que solucionaban distintas circunstancias como materiales, formas, tecnologías, cromáticas, funciones, además de la viabilidad de venta de un producto de esta índole. Seguidamente, se respaldó la información resultante al momento, por medio de entrevistas a cinco usuarios potenciales. 


\section{Definiendo la funcionalidad}

En esta segunda etapa, con el uso de un árbol de funciones, se delimitaron las funciones que tendría el sistema y la manera en la que se iban a distribuir entre los dos productos complementarios. Además, se propusieron distintas combinaciones de soluciones por medio de una matriz morfológica.

Tras tener claras las funciones, se procedió a investigar la disponibilidad en el mercado tanto de materiales para su construcción como de componentes electrónicos o mecánicos que ayudarán a cumplir las funciones que fueran estándares. Luego se determinaron las partes que compondrían a los productos por medio de un árbol de arquitectura de producto ${ }^{1}$ (figura 2) y se definió el comportamiento de los productos ante distintas situaciones con un diagrama de flujo. Finalmente, se propusieron algunas ideas rápidas por medio de bocetos.

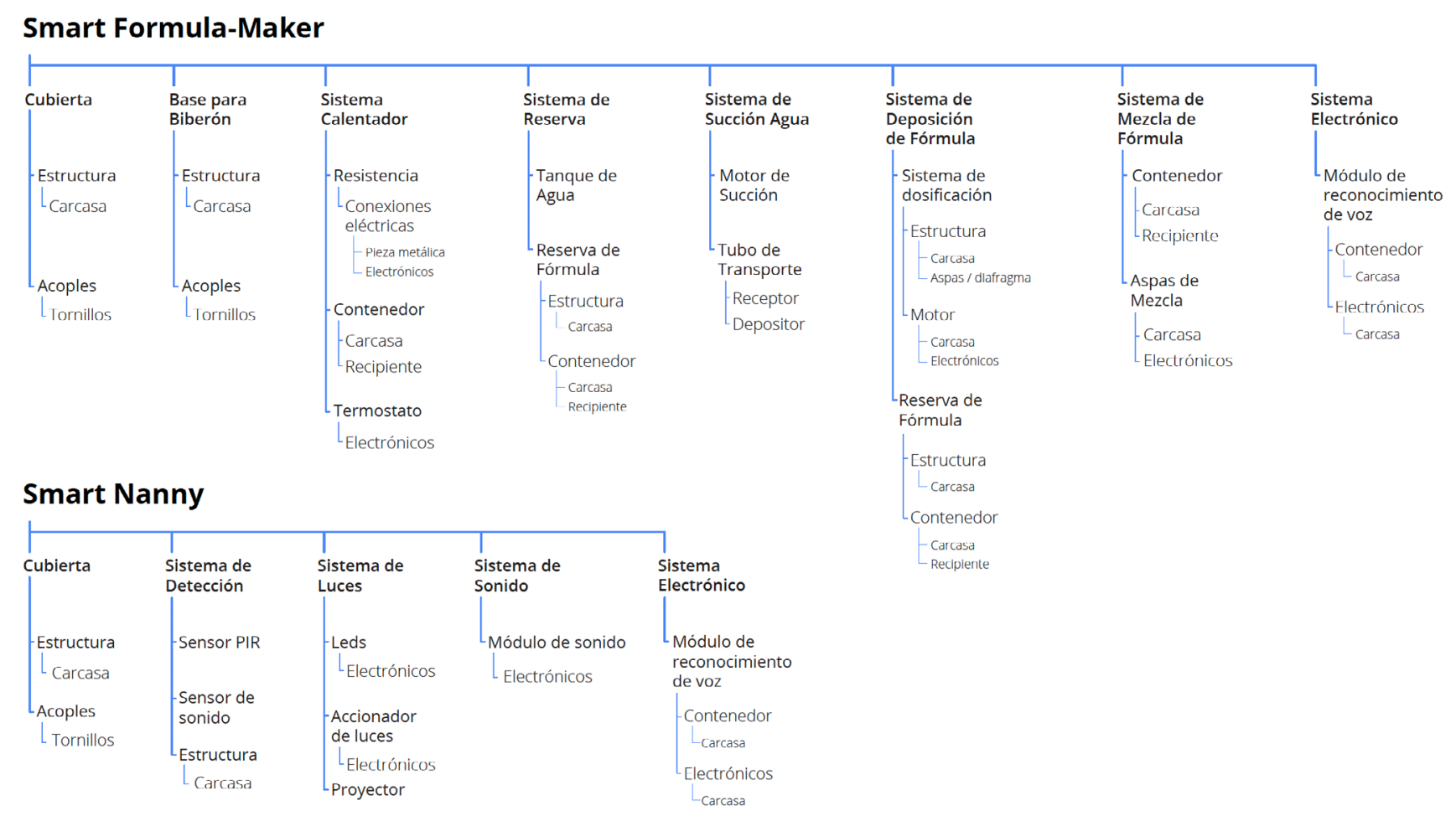

Figura 2. Árboles de arquitectura de los productos.

Fuente: elaboración propia, 2019.

1 Herramienta que se basa en hallar la relación existente entre un concepto general (producto) y los elementos que lo componen (partes o sistemas) a través de un proceso sistemático. 


\section{Definiendo la forma}

La tercera sección del proyecto se comenzó al definir un concepto de diseño, así como sus parámetros, objetivo general, objetivos específicos, alcances y expectativas, además, se respondieron las "WWWWWH" (what, why, when, where, who, how); posterior a esto y teniendo muy presentes los parámetros del concepto, se procedió a definir la perceptualidad del producto con el uso de ejes semánticos, moodboards, matrices cromáticas y análisis morfológicos de los ejes; con esto claro, se desarrollaron abundantes propuestas gráficas (esta vez, dibujos con una calidad mucho mayor, al menos seis propuestas por cada producto), en las cuales se buscó explotar los hallazgos de esta etapa y todas las posibles soluciones de interacción, morfología y cromática.

Luego se evaluaron las propuestas con respecto a la tabla de requisitos de la primera etapa y se seleccionó la propuesta con el mayor puntaje; esta se ilustró en un storyboard en el que se explicaba su funcionamiento y su interacción. Para cerrar la etapa, se realizó una validación perceptual por medio de un cuestionario de tipo diferencial semántico. El diferencial semántico se le aplicó individualmente a un grupo de 15 padres primerizos con bebés menores a 18 meses que cumplen las características del usuario definido en la etapa 1.

\section{Detallando la manufactura}

La cuarta etapa abarcó todos los temas técnicos y de logística, ya que se trataba de construir el cuaderno técnico del producto. Acá se explicaron los aspectos generales: "cómo funciona" y "cómo se usa", además de abarcar cómo se comporta el sistema y qué aporta cada uno de los productos a este. Fue necesario modelar en 3D todas las partes que componen a los productos (ver figura 2) para, posteriormente, hacer explosos de cada producto, así como los planos para cada pieza. Se determinó el material de cada una de las piezas para definir la manera en la que se manufacturarían y determinar qué componentes estándar se usarían para identificar la mejor manera de ensamble, así como los tipos de ensambles que se implementarían.

Para las medidas del sistema y las interfaces tangibles (como las manijas de los compartimentos y el tanque de agua) con las que interactúan los usuarios, se tomaron en cuenta medidas antropométricas de los posibles usuarios y medidas estándar en la industria. Posteriormente, se mostraron los diagramas electrónicos, es decir, la manera en la que se conectan los circuitos y componentes electrónicos, y se adjuntaron a la programación realizada. Luego, se estipularon los costos con base en lo que se había gastado. Finalmente, se agregaron las instrucciones de uso de los objetos y la limpieza del sistema.

\section{Validando el producto}

Para la quinta etapa, se comprobó que los resultados obtenidos fueran correctos; por esto, se acude a pruebas con usuarios reales. Estos usuarios tienen bebés menores a 18 meses, los alimentan con fórmula materna y han hecho uso de tecnologías inteligentes anteriormente. Con 
ellos se realizaron evaluaciones de funcionalidad, usabilidad y perceptualidad. Se diseñaron las siguientes herramientas: un guion y tareas por realizar para hacer "ensayos con usuarios", con esto se busca probar la funcionalidad del producto al estudiar cómo se comportaba el tester frente al objeto; entrevistas para evaluar la usabilidad, se plantearon preguntas que contemplaron los cinco aspectos de la usabilidad (efficiency, learnability, memorability, error, and satisfaction), además, los datos de la entrevista se triangularon con los de la observación para tener un resultado más fidedigno. Finalmente, se aplica un cuestionario con preguntas de tipo diferencial semántico para evaluar en una escala cuán amigable, moderno, su connotación de electrodoméstico, funcional y agradable [6]. Esta se ejecutó con siete usuarios potenciales y sus resultados se analizaron y sintetizaron, además, se extrajeron las conclusiones.

\section{Resultados}

Cada etapa cierra con hallazgos sobresalientes y estos permitieron moldear el rumbo del proyecto.

En Definiendo la funcionalidad, se determina cuál era la necesidad que se ve satisfecha con el sistema, así como una delimitación clara de este (ver figura 3).

\section{Necesidad}

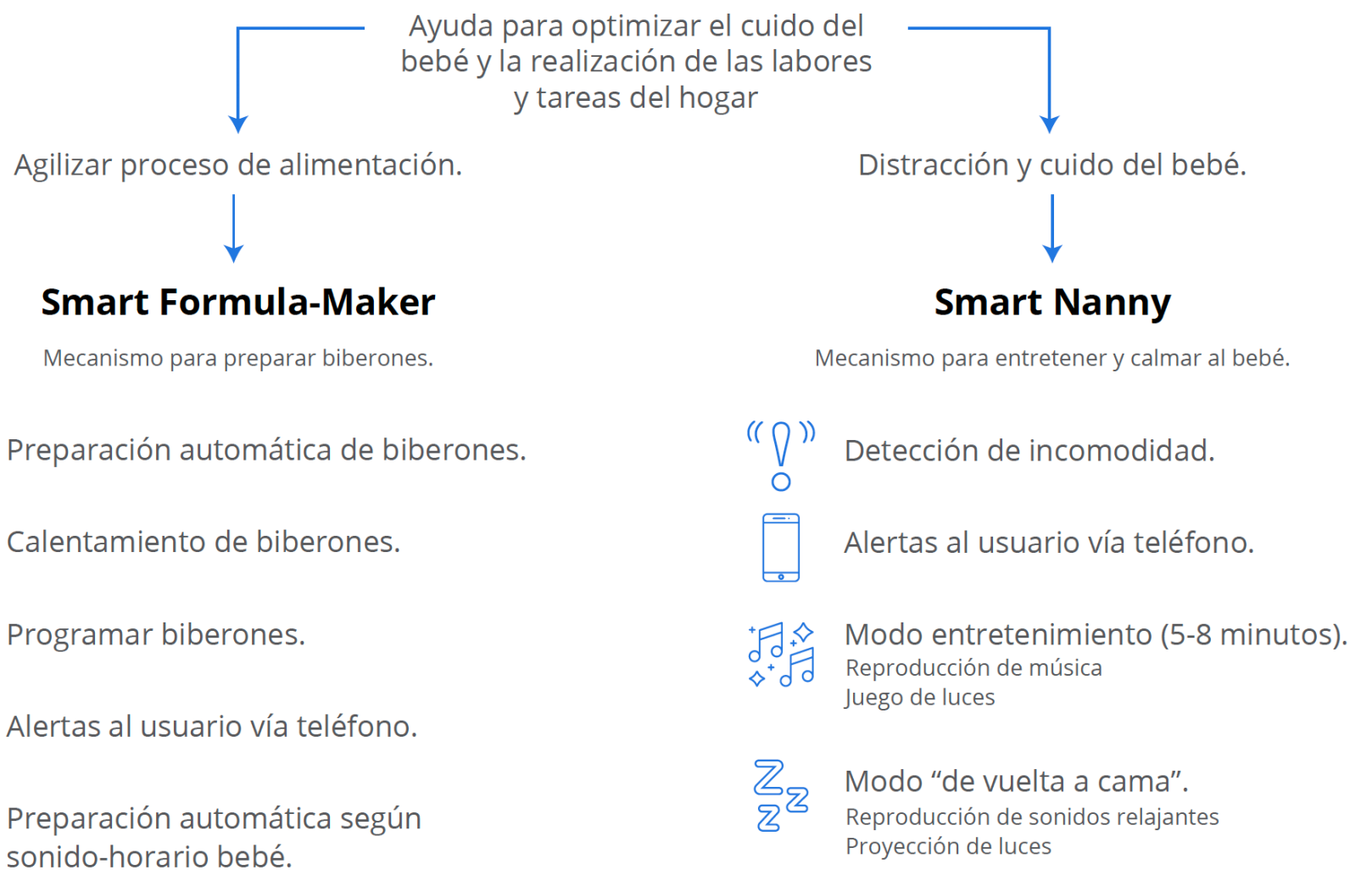

Figura 3. Mapa conceptual de la definición del sistema.

Fuente: elaboración propia, 2019. 
Una vez que las necesidades fueron esclarecidas, se delimitan las funciones tanto analógicas como inteligentes para el SFM, se considera que debe poder detectar la temperatura, calentar agua, mezclar los ingredientes y otros; mientras que para el SN, se considera que permita la reproducción de sonidos, emitir luces, detectar movimiento y otras. Con estas presentes, se investiga la disponibilidad en el mercado para conseguir las piezas mecánicas o componentes electrónicos que se definen en la siguiente etapa.

Para Definiendo la forma, se define el concepto. Este se basa en las necesidades recopiladas al inicio y la independencia que necesitan los padres en la vida moderna; por eso se determina como Independencia moderna el concepto. Este comprendido como la cualidad de un objeto de tener autonomía por medio de funciones inteligentes que le permitan brindar libertad al usuario.

Esta definición se expresa también contemplando al usuario, la interacción usuario-objeto, el entorno, su perceptualidad y su forma. Además de delimitar los alcances del proyecto:

- Prototipar un asistente inteligente que pueda hacer biberones de fórmula automáticamente y otro que distraiga al niño.

- Realizar una interfaz oral entre el sistema y el usuario.

- Desarrollar un sistema de productos que puedan comunicarse entre sí.

Seguidamente, con el análisis cromático y morfológico (ver figura 4), se determina:

\section{Cromática}

- Se usarán colores neutros, en especial el blanco. Esto con el objetivo de darle un aspecto minimalista y limpio.

- Se utiliza el negro para contrastar ciertas áreas de interacción ("sensores") y el gris como complemento.

- Colores pastel con una baja saturación y alta luminosidad.

- Al utilizar metal se puede dejar su color natural para crear un aspecto más moderno o tecnológico.

\section{Morfología}

- Formas circulares, esféricas y ovaladas. Esto contribuye a la perceptualidad de ver el objeto "amigable".

- $\quad$ No posee esquinas puntiagudas ni ángulos rectos.

- $\quad$ Alta continuidad de superficies.

- Tamaño pequeño-mediano y una forma compacta para incomodar lo menos posible al usuario.

- Componentes desprendibles para facilitar usabilidad y la higiene.

- Los objetos con una "cara" simple y feliz se perciben más amigables. 


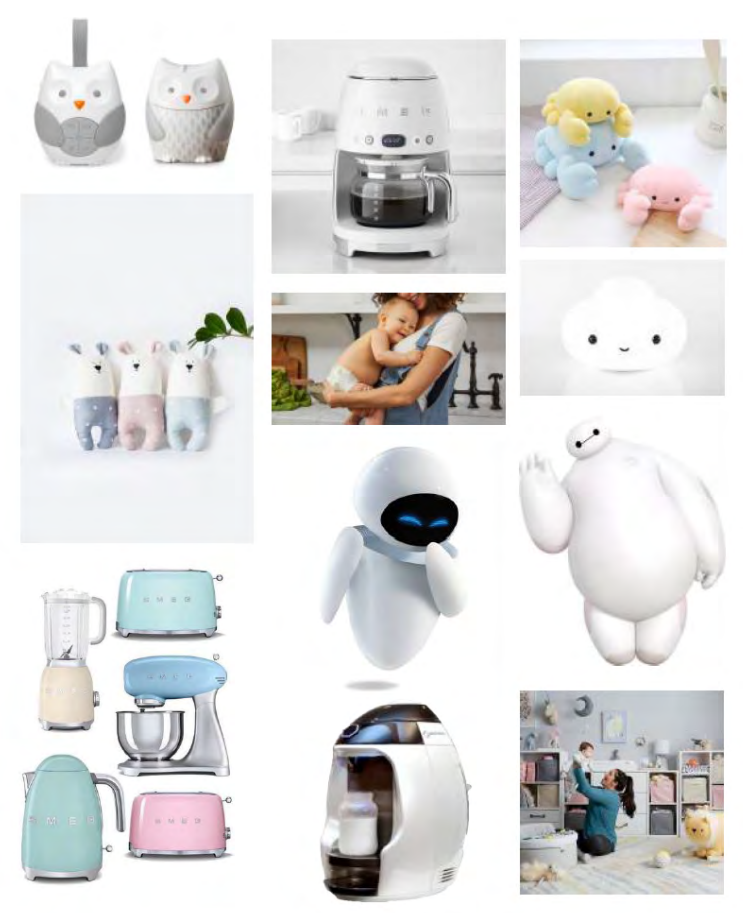

Figura 4. Moodboard del análisis morfológico y cromático.

Fuente: elaboración propia, 2019.

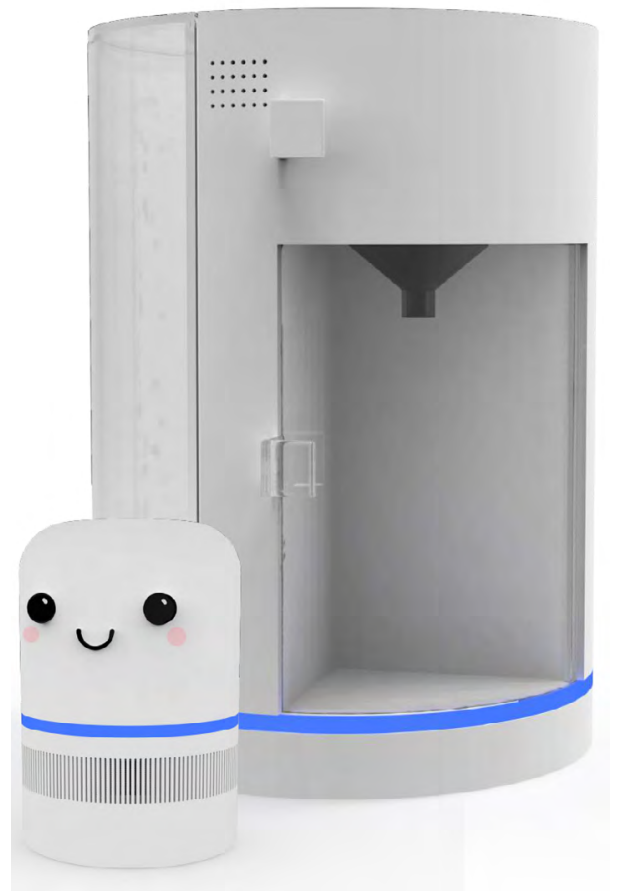

Figura 5. Render del sistema MiA.

Fuente: elaboración propia, 2019. 
En la figura 5, se observa el resultado final de la etapa 4, Detallando la manufactura, se puede ver cómo este encaja adecuadamente en el moodboard de la figura 4, lo que indica que la perceptualidad se aplicó de manera correcta.

En la etapa 4, se definen los materiales para los productos: ABS y Copoliéster EX401, por su resistencia a impactos (en caso de entrar en contacto con el bebé) e higiene, respectivamente [7], esto porque, al ser productos que involucran a un infante, deben estar libres de bacterias o agentes infecciosos.

Los componentes estándar que se definen son:

Smart Formula Maker

Acople de resistencia, resistencia monofásica con una varilla forma ' $U$ ', abrazadera de bomba, manguera de silicona, difusores, Shield EasyVR 3.0, motor rotatorio, wireless transceiver module, bomba de agua y contactless temperature sensor module.

Smart Nanny

DF player mini, neopixel, ESP8266, thin speaker COM-10772, wireless transceiver module, difusores, $\mathrm{KY}-038$, sensor de movimiento.

Seguido a esto, se plantea una estrategia de manufactura (ver figura 6) en la que se aborda el flujo de producción, así como los ensambles que se usan para los productos.

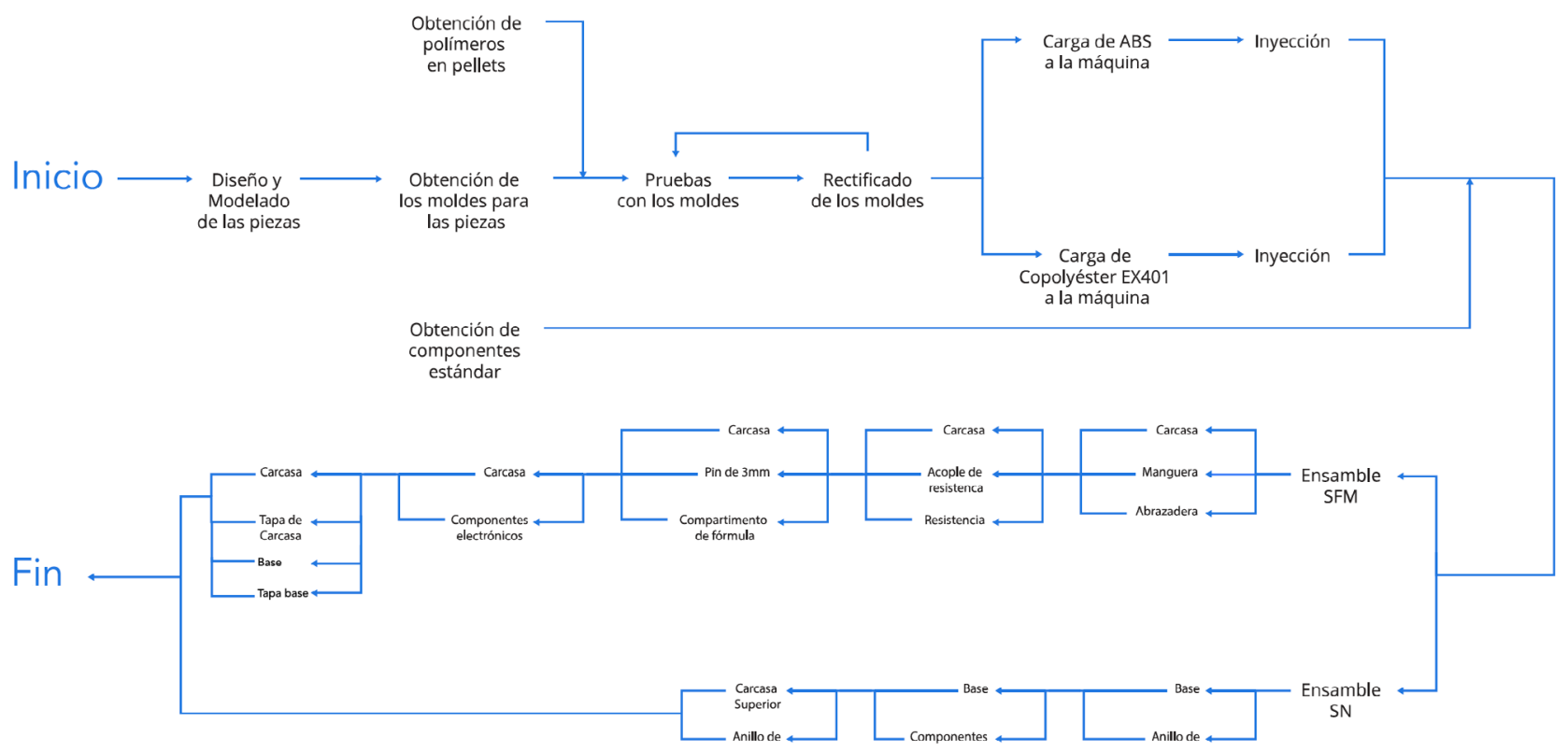

Figura 6. Flujograma de manufactura del sistema MiA.

Fuente: elaboración propia, 2019. 
También se desarrolló un instructivo o manual de uso como apoyo para los usuarios. Principalmente para demostrar el proceso de limpieza diaria y mensual del Smart Formula Maker, este aspecto es importante debido a la naturaleza delicada de los bebés y su alimentación. También se definen requerimientos para el funcionamiento correcto del Smart Nanny y el uso general del sistema.

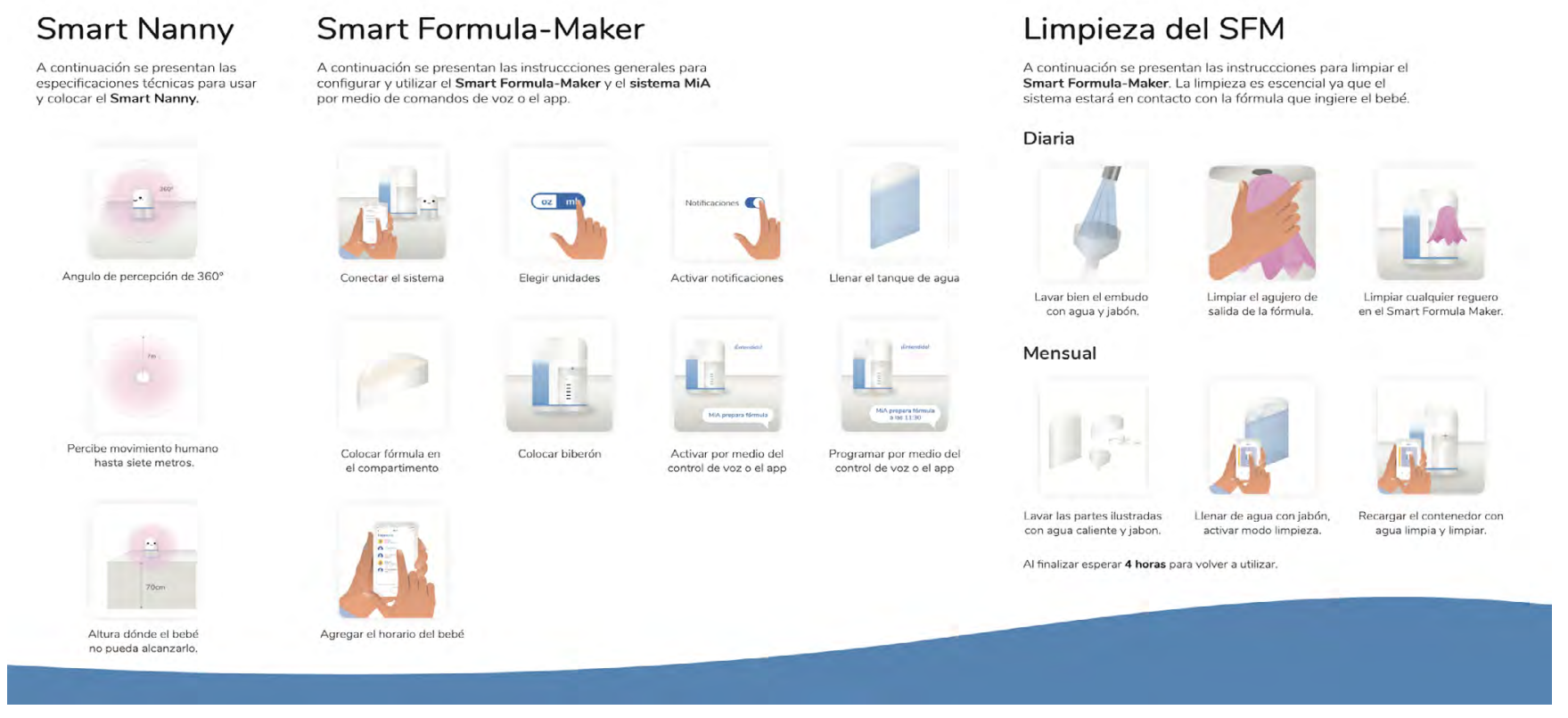

Figura 7. Instructivo para el uso del sistema MiA.

Fuente: elaboración propia, 2019.

Finalmente, en la etapa Validando el producto, tras realizar las pruebas, se repiten algunos patrones y estos dan lugar a los siguientes hallazgos; en términos de la configuración del sistema, la interfaz física es bastante intuitiva, sin embargo, en cuanto a la funcionalidad, al usuario le gustaría poder indicarle al SFM cuántas onzas quiere preparar en su biberón.

En cuanto a preparación automática, hay confusión, al existir muchas maneras de cómo pedir fórmula; mientras que el SFM no entendió la mayoría (esto debido a limitaciones de prototipo), este problema suscita también para programar un biberón con antelación. Se propone como solución a esto incluir palabras clave en el instructivo con el fin de limitar la posibilidad de error.

Los usuarios sugieren que el $\mathrm{SN}$ debe tener mayor versatilidad en las canciones del modo entretenimiento.

En términos de usabilidad, se define que el usuario sí debe aprobar cualquier acción que haría el SFM, además, que tenga mayor capacidad para no tener que rellenar diariamente el objeto. Así mismo, sugieren incluir una luz de estado que ayudará a indicarle al usuario si lo está escuchando. 


\section{Perceptualidad}

\author{
Simple
}

Amigable

Moderno

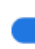

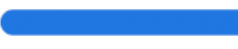

$83 \%$

Agradable
$92 \%$

$92 \%$

$92 \%$

Figura 8. Gráfico de perceptualidad del sistema MiA.

Fuente: elaboración propia, 2019.

A raíz de esto, se evidencia que, para investigaciones futuras, se debe considerar el estudio de productos que ya utilicen reconocimiento por voz y su usabilidad, con el fin de facilitar el entendimiento usuario-objeto, métodos para ampliar la cantidad y versatilidad de canciones dentro del SN; asimismo, se debe investigar sobre un sistema de confirmación del usuario para cualquier acción que vayan a realizar los productos, esto con el fin de facilitar el uso del sistema para los usuarios y que este sea más eficiente.

\section{Conclusiones}

El desarrollo de este sistema de productos inteligentes permite ver el diseño desde áreas distintas, ampliando el conocimiento y pensamiento crítico para resolver problemas, por ejemplo, en el ámbito de electrónica, se logra comprender el funcionamiento de los componentes utilizados. El mezclar estas dos disciplinas permite responder a las necesidades del usuario de una manera más personalizada. Además, permite crear productos más tecnológicos y con diversas funciones, los cuales son los más utilizados por los usuarios hoy en día [8].

El diseño, al ser una disciplina multidisciplinaria e integral, permite generar diversos resultados al combinarse con diferentes ciencias. Para el caso de este proyecto, se integró con la electrónica y esto permitió el desarrollo de un sistema inteligente que reduce el trabajo manual, por ende, el tiempo de las tareas del usuario. Sin embargo, puede colaborar con cualquier otra rama y obtener un producto muy diferente, pero igualmente valioso.

Se emplean técnicas aprendidas para validar el sistema y realizar sus respectivas mejoras con el fin de cubrir las necesidades encontradas a la hora de conversar con usuarios y analizar su problemática. Por estas razones, se define un producto concreto y funcional con el cual se logra satisfacer a los usuarios. 
En un futuro, se pueden realizar más pruebas con usuarios, con el objetivo de encontrar oportunidades de mejora y al integrarlas, generar un prototipo de alta fidelidad.

\section{Referencias bibliográficas}

[1] INEC-Costa Rica, "Nacimientos", 2018. Disponible en: http://inec.cr/poblacion/ nacimientos.

[2] K. Basire, S. Pullon, and D. McLeod, "Baby feeding: the thoughts behind the statistics", vol. 110, no. 1044, p. 184-187, May 1997. [Online]. Disponible en: http://europepmc.org/ abstract/MED/9201204.

[3] Gibbs, "How smart speakers stole the show from smartphones", 2018. [Online]. Disponible en: https://www.theguardian.com/technology/2018/jan/06/how-smartspeakers-stole-the-show-from-smartphones.

[4] V. Sandulescu, "Designing Assistive Technology for Domotic Systems", vol. 7, no. 4, 2015, [Online]. Disponible en: https://www.scientificbulletin.upb.ro/rev_docs_arhiva/ fulla7a_559847.pdf.

[5] J. Craven, "Exploring Home Automation and Domotics", Feb. 11, 2020. Disponible en: https://www.thoughtco.com/what-is-a-smart-house-domotics-177572 (accessed Jun. 07, 2020).

[6] A. Milton and P. Rodgers, "Métodos de investigación para el diseño de producto". Barcelona, España: Blume, 2013.

[7] “Tritan Copolyester EX401", 2010. Disponible en: https:/www.eastman.com/products/ pages/producthome.aspx?product=71068690 (accessed Jun. 07, 2020).

[8] Á. González, "Amazon Lets Users Create Their Own Smart Home”, Apr. 06, 2016. Disponible en: https://www.govtech.com/products/Amazon-Lets-Users-Create-TheirOwn-Smart-Home.html (accessed Jun. 07, 2020). 